\title{
BOOK REPORT
}

\section{Critical Care Medicine: The Essentials}

\author{
Daniel De Backer* \\ Critical Care Medicine: The Essentials. 4th edition. Edited by: Marini JJ, Wheeler AP. Wolters Kluwer/Lippincott Williams and \\ Wilkins, 2010. ISBN 978-0-7817-9839-6
}

Critical Care Medicine: The Essentials is already in its fourth edition. What are the essentials? The essentials are a mixture of sound physiology and ground knowledge on diseases and established therapies.

Why should we read The Essentials? All patients do not behave the same, therefore physicians have to decide patient management using clinical judgment based on clinical signs, on core physiology and on patient response to disease and interventions. Most of the attractiveness of this book comes from the fact that it is written by clinicians for clinicians. It describes a lot of clinical signs, (patho)physiological patterns, X-ray aspects, and other small tricks that guide the physician to rapidly diagnose at the bedside what the patient may present or to better set up ventilatory support.

This book is separated into two parts: the first relates to techniques and methods, while the second relates to diseases. The separation is sometimes artifactual, as mechanical ventilation has to be discussed as a technique but also for the management of ventilatory failure. Nevertheless, this separation does not impede reading because there is no overlap and because this book is not a handbook. Chapters have to be read cautiously, taking time to understand the details of physiological principles or the interaction between disease processes and interventions.

The book covers most aspects of intensive care practice, including a rapid description of the diseases that may be encountered in critically ill patients, the specificities of critically ill patients, and the therapeutic interventions and monitoring techniques that may be used. The quality of the different sections is quite heterogeneous. The cardiovascular sections are excellent and the respiratory sections are really astonishing. Even physicians with excellent knowledge in respiratory medicine will learn by reading these parts. The sections describe all types of respiratory failure and discuss their specific management. Respiratory patterns and ventilatory support are reported extensively.

The section on hemodynamic monitoring is attractive including a large part on the interpretation of pulmonary artery tracings (which is particularly useful in present times when physicians are more reluctant to insert pulmonary artery catheters and may sometimes lack some of the basic knowledge required to interpret the tracings), but also excellent parts related to non-invasive monitoring including echocardiography.

Imaging techniques are well reported, with a lot of attention paid to visualization of lines and tubings. One can nevertheless regret that noncardiac echography is not discussed. The chapters on analysis of acid-base status and interpretation of blood gas and electrolyte disorders are also very good.

Some chapters are definitively weaker. The chapters on renal epuration, especially hemofiltration, and on sepsis are oversimplified.

The book also includes several appendices, including physiological equations (mostly respiratory and cardiovascular formulas) and some physical correction factors.

In summary, Critical Care Medicine: The Essentials is very attractive for physicians involved in the care of critically ill patients and for physicians who need to train or to perfect their knowledge, especially in the field of thoracic and cardiovascular medicine.

Published: 9 August 2010

doi:10.1186/cc9204

Cite this article as: De Backer D: Critical care medicine: the essentials. Critical Care 2010, 14:3??. 